\title{
GLAD!
}

Revue sur le langage, le genre, les sexualités

$01 \mid 2016$

Varia

\section{Genre et migrations}

L'autonomie à l'épreuve du volet linguistique du contrat d'accueil et d'intégration

Gender and Migrations. The Impact of the Accomodation and Integration

Contract's Linguistic Requirements on Autonomy

\section{Nadia Ouabdelmoumen}

\section{OpenEdition}

Journals

Édition électronique

URL : http://journals.openedition.org/glad/197

DOI : $10.4000 /$ glad. 197

ISSN : 2551-0819

Éditeur

Association GSL

Référence électronique

Nadia Ouabdelmoumen, «Genre et migrations », GLAD! [En ligne], 01 | 2016, mis en ligne le 15 novembre 2016, consulté le 17 décembre 2020. URL : http://journals.openedition.org/glad/197 ; DOI https://doi.org/10.4000/glad.197

Ce document a été généré automatiquement le 17 décembre 2020.

\section{c) (7) $\Theta$}

La revue GLAD! est mise à disposition selon les termes de la Licence Creative Commons Attribution Pas d'Utilisation Commerciale - Pas de Modification 4.0 International. 


\title{
Genre et migrations
}

\author{
L'autonomie à l'épreuve du volet linguistique du contrat d'accueil et \\ d'intégration \\ Gender and Migrations. The Impact of the Accomodation and Integration \\ Contract's Linguistic Requirements on Autonomy
}

\section{Nadia Ouabdelmoumen}

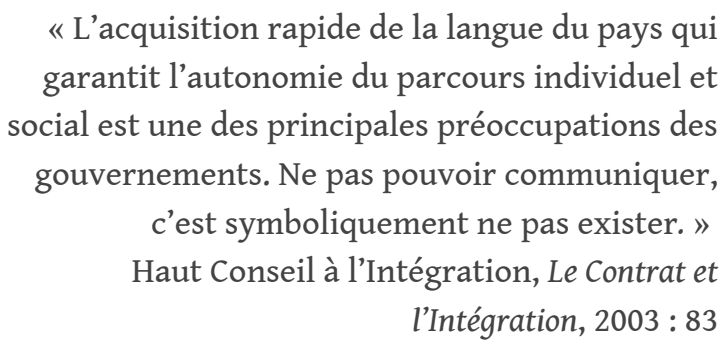

L'autonomie est un idéal central des sociétés dites modernes. Dans un premier sens, elle désigne le processus par lequel un groupe social ou un individu se gouverne par ses propres lois ou selon ses propres intérêts. Son développement est concomitant à l'avènement théorique de l'individu au XVIII siècle, puis de l'individualisme, de la responsabilisation, des nouvelles formes de division du travail, de la spécialisation et de l'interdépendance. Durant le $\mathrm{xx}^{\mathrm{e}}$ siècle, l'histoire des mouvements ouvriers et de l'éducation populaire fera de l'autonomie de l'enfant un objectif pédagogique majeur. De leur côté, les mouvements de 1968 érigeront cet idéal comme une force libératrice susceptible de contrer les inégalités sociales, notamment celles induites par le patriarcat, dans un contexte de dénaturalisation et de politisation des rapports sociaux de sexe (Delphy 2001). Au centre des revendications d'émancipation collective et individuelle dans les années 1960 et 1970, cet idéal se trouve cependant aujourd'hui critiqué pour sa potentielle fonction de légitimation de l'ordre social. Généralement, les débats actuels s'accordent pour y voir « une certaine version de la liberté caractérisée par un rapport cognitif et pratique à soi privilégié » (Jouan 2012 : 43). Mais, à l'instar de Foessel pour qui il «n'existe pas de moyen plus efficace de contraindre les individus que celui qui les convainc qu'ils agissent librement » $(2011: 345)$, cet idéal des Lumières peut également être perçu comme un instrument de contrôle et une façon de 
gouverner les individus « depuis leur propre liberté » proclamée. En outre, en devenant un objectif performatif des politiques publiques, ce poncif de l'action sociale (Alberola \& Dubéchot 2012 : 149) se serait transformé en une sorte de rhétorique des institutions.

En partant d'une recherche de doctorat qui a consisté à interroger l'actualisation du genre et des rapports sociaux dans le cadre de la mise en application du volet linguistique du contrat d'accueil et d'intégration à Rennes (France) ${ }^{1}$, cette contribution vise à interroger l'autonomie comme un principe sur lequel, et par lequel, se fonde ce dispositif contractuel de l'offre-obligation de formation linguistique. Après avoir défini les préalables théoriques relatifs à notre questionnement: le genre et les rapports sociaux, et avoir présenté le contrat d'accueil et d'intégration (CAI) et son volet linguistique (VL), nous décrirons la manière dont apparait, sur ce terrain d'enquête, une forme d'injonction à l'autonomie par la langue qui s'adresse prioritairement aux femmes stagiaires de ce dispositif. Cela, en veillant à croiser les discours de (sur) l'autonomie avec une interprétation des conditions sociohistoriques, politiques, etc. qui participent à leur production et à la réactualisation des rapports sociaux. Sans omettre que les dimensions de classe traversent les phénomènes sociaux décrits, notre analyse se centrera davantage sur l'articulation-actualisation du genre et de la race.

3 La posture méthodologique adoptée est interdépendante d'une posture théorique de départ, elle-même liée à la définition du genre. En effet, dans cette recherche, le genre est considéré comme un rapport social fondé sur des processus de différenciation et de hiérarchisation entre les sexes (Scott 1988: 141) et des processus, notamment discursifs, de fabrication et d'institution des sexes. Prenant acte des critiques adressées à l'hégémonie d'une pensée féministe révélant un usage raciste de la catégorie de genre (black feminism, post colonial studies, subaltern studies), aux théories traitant du sexisme comme contradiction secondaire des sociétés de classe capitaliste et enfin, aux approches privilégiant un pluralisme cumulatif des rapports de domination (intersectionnalité), les rapports sociaux sont envisagés ici en termes de consubstantialité et de coextensivité. Dans ce cadre, la notion de rapport social désigne "une relation antagonique entre deux groupes sociaux, établie autour d'un enjeu " (Kergoat 2009: 112) et/ou une "tension qui traverse le champ social et qui érige certains phénomènes sociaux en enjeux autour desquels se constituent des groupes sociaux aux intérêts antagoniques » (Pfefferkorn 2012: 95-96). Et, puisque le genre est un rapport social parmi d'autres, les rapports sociaux de sexe, de race et de classe sont envisagés comme consubstantiels, car ils « forment un nœud qui ne peut être séquencé au niveau des pratiques sociales » et coextensifs puisqu'ils "se produisent et se reproduisent mutuellement en se déployant » (Kergoat 2009 : 112).

4 Ces préalables sont au centre de la manière dont a été abordé le protocole d'enquête (de 2009 à 2011).

\section{Un protocole d'enquête articulé autour de l'observation directe et l'entretien semi-directif}

5 La phase d'observation directe s'est déroulée dans deux organismes de formation et a concerné quatre dispositifs de formation linguistique (FL) à destination des personnes immigré-e-s sur le territoire rennais. Dans un premier organisme, ces observations ont concerné les Ateliers de Savoirs Sociolinguistiques (ASL) et les plateformes d'évaluation 
et d'orientation de la demande de formation linguistique ${ }^{2}$. Dans un second organisme de formation, en charge de la prescription linguistique CAI à Rennes, ces observations ont concerné les plateformes d'accueil des stagiaires CAI et les formations linguistiques CAI et Hors-CAI ${ }^{3}$, à l'occasion de dix-sept séances de préparation à la passation du DILF (Diplôme Initial de Langue Française)/DELF (Diplôme d'Études en Langue Française), dans six lieux de formation sur le territoire rennais.

6 La phase d'entretien semi-directif a été menée dans l'organisme agréé pour la FL CAI à Rennes, auprès de cinq enseignantes $\mathrm{CAI}$ et de quatorze signataires-stagiaires CAI et hors-CAI.

7 À l'époque du déroulement de ces enquêtes de terrain, l'équipe de formation CAI est exclusivement constituée d'enseignantes.

\section{L'égalité entre les sexes et l'autonomie : au cœur de la mise en place du volet linguistique du contrat d'accueil et d'intégration}

8 Le contrat d'accueil et d'intégration est un document individuel signé entre l'État et toute personne étrangère "non communautaire » admise "pour la première fois au séjour (...) ou qui entre régulièrement en France (...) et qui souhaite s'y maintenir durablement $»^{4}$.

9 La signature de ce contrat qui peut intervenir en amont de l'émigration, à travers le pré-CAI, et auquel peut s'ajouter la signature d'un Contrat d'Accueil et d'Intégration pour la Famille (CAIF), engage les personnes contractantes à participer à une session d'information sur la vie en France, à un bilan de compétences professionnelles pour les signataires non titulaires d'un emploi, à une formation civique et, enfin, le contrat d'accueil et d'intégration implique un volet de prescription linguistique obligatoire depuis le $1^{\text {er }}$ janvier 2007, suite à la loi du 24 juillet 2006 relative à l'immigration et à l'intégration.

10 Dans ce cadre, toute personne qui désire rejoindre la France, dans le cadre du regroupement familial, de même que les conjoint-e-s étranger-ère-s de Français sollicitant un visa de long séjour, doivent faire l'objet d'une évaluation permettant d'apprécier leur degré de connaissance de la langue française. Si le besoin en est établi, une formation linguistique leur est délivrée. Dans ce cas, et à l'issue d'environ 400 heures de formation, les signataires-stagiaires du contrat d'accueil et d'intégration sont soumis-e-s à une évaluation que sanctionnent soit le DILF, soit le DELF A1 à partir de 2010.

\section{"Agissons pour que les droits de la femme française s'appliquent aussi aux femmes de l'immigration » : un contrat pour l'égalité entre les sexes}

Dès 2003, lorsque le contrat d'accueil et d'intégration est expérimenté dans quelques départements français, un lien explicite est établi entre sa nécessité et l'importance que revêt le principe de l'égalité entre les femmes et les hommes. Cette mise en corrélation est perceptible dans de nombreux discours politiques qui accompagnent sa mise en place, 
dont cet extrait d'allocution de Sarkozy, alors ministre de l'Intérieur, à l'occasion d'une convention intitulée Une immigration choisie, une intégration réussie, le 9 juin 2005 :

Il faut être plus volontariste et exigeant en matière d'accueil et d'intégration. Le contrat d'accueil doit être obligatoire et le contenu des formations plus dense. Nous sommes fiers des valeurs de la République, de l'égalité entre les hommes et les femmes, de la laïcité, de l'idéal français d'intégration. Alors, osons en parler à ceux que nous accueillons. Et agissons pour que les droits de la femme française s'appliquent aussi aux femmes de l'immigration ${ }^{5}$.

D’ailleurs, dans le contrat présenté à la signature, l'égalité est exclusivement définie comme une égalité entre les sexes :

L'égalité entre les hommes et les femmes est un principe fondamental de la société française. Les femmes ont les mêmes droits et les mêmes devoirs que les hommes. Les parents sont conjointement responsables de leurs enfants. Ce principe s'applique à tous, Français et étrangers. Les femmes ne sont soumises ni à l'autorité $\mathrm{du}$ mari ni à celle du père ou du frère pour, par exemple, travailler, sortir ou ouvrir un compte bancaire. Les mariages forcés et la polygamie sont interdits, tandis que l'intégrité du corps est protégée par la loi ${ }^{6}$.

Le contrat d'accueil et d'intégration et son volet linguistique sont mis en place dans un contexte de restriction de l'immigration adossée à une rhétorique des besoins économiques. À l'occasion de cette mise en opposition de l'immigration, entre immigration choisie - c'est-à-dire immigration de travail, de talents et de compétences - et immigration subie - c'est-à-dire immigration familiale et/ou illégale, c'est notamment au nom de l'attachement à l'égalité entre les sexes que parait légitimé le contrat d'accueil et d'intégration comme une sorte de kit contractuel, garant d'une intégration réussie, parce que choisie (Fassin 2006).

\section{L'autonomie : un principe inhérent au soubassement contractuel du CAI}

En outre, l'apparition et la mise en application du CAI et de son volet linguistique sont à situer dans deux autres contextes permettant d'interpréter les discours associés à l'autonomie dans le cadre de nos enquêtes. En effet, la mise en fonctionnement de ce dispositif des politiques de l'immigration et de l'intégration est concomitante à une multiplication des formes contractuelles d'action publique depuis une trentaine d'années (Gaudin 2007) et de prolifération de processus de suivi individualisés et contractualisés, privilégiant des mécanismes capacitants de responsabilisation individuelle, de valorisation de l'autonomie, de socialisation par le travail et de lutte contre l'inactivité, dans un contexte paradoxal marqué par une pénurie de l'emploi. Enfin, ce dispositif apparait dans un contexte de généralisation des rhétoriques de l'insécurité, justifiant le renforcement d'instruments et d'actions de contrôle et de sécurité institutionnalisés (Bouquet $2012: 18$ ).

L'incitation à la responsabilité et à l'autonomie est inhérente au soubassement contractuel du VL du CAI, ce qui en fait un cadre interprétatif non négligeable pour la compréhension de l'autonomie, un principe qui est éminemment lié à la connaissance de la langue, considérée comme un «facteur essentiel d'autonomie économique, sociale et culturelle» ou comme «un vecteur essentiel d'intégration et une condition nécessaire et indispensable pour accéder rapidement à l'autonomie $»^{7}$. 
Dans ce contexte prescriptif, la connaissance-maitrise de la langue française est envisagée comme une nécessité pour l'autonomie individuelle, cette dernière étant pensée comme une exigence face aux risques de chômage, de précarité, d'inégalité entre les sexes, etc. Plus encore, la langue elle-même est désignée comme «langue de l'autonomie » et son apprentissage décrit comme devant inclure " par le vocabulaire, la syntaxe et leurs usages » entre autres valeurs et principes « l'égalité des hommes et des femmes $»^{8}$.

17 Aussi, considérant que les langues s'inscrivent dans un ensemble de processus qui légitiment ou délégitiment les pratiques sociales (Canut \& Duchêne 2011:5) et qu'elles participent à l'actualisation du caractère hiérarchisé et hiérarchisant de ces pratiques sociales, soit des rapports sociaux, la suite de notre propos visera à décrire la manière dont apparait, dans le cadre ce dispositif de contrôle et de sécurité (Foucault 2004 : 50), une forme d'exigence différenciée à l'autonomie, révélatrice de la prégnance du contrôle social, entendue comme un "corpus d'exigences normatives de régulation sociale» (Amyot 2012: 127) dont le but est d'assurer une forme d'homogénéité des comportements sociaux, dans une visée de cohésion sociale.

\section{Autonomie par la langue et actualisation du genre et des rapports sociaux dans le cadre du volet linguistique du contrat d'accueil et d'intégration}

\section{L'autonomie : un objectif central et transversal du contrat d'accueil et d'intégration et de son volet linguistique}

L'autonomie ne figure pas parmi les notions explicitement interrogées lors des enquêtes de terrain et aucun-e stagiaire ne la mentionne lors de nos échanges. Néanmoins, cette notion est particulièrement mobilisée par les enseignantes en charge de la prescription linguistique CAI, lorsqu'elles évoquent leurs attentes vis-à-vis des stagiaires et/ou l'intérêt que représentent la formation linguistique et le DILF/DELF pour ces dernier-ère-s. Ce principe n'est pas directement mis en lien avec le projet pédagogique que sous-tend le volet linguistique du CAI. Elle n'est pas évoquée pour interroger le degré d'encadrement des stagiaires, leur capacité à effectuer des tâches sans se référer aux enseignantes et/ou pour désigner la capacité des apprenant-e-s à s'autonomiser dans leur parcours de formation, selon une logique d'efficacité des apprentissages. Le plus souvent, l'autonomie est décrite comme un objectif final et transversal de ce dispositif à destination des immigré-e-s non-communautaires. Dans ce cadre, la langue est plébiscitée comme pilier de cette autonomie sociale, un aboutissement qui passe nécessairement par l'autonomie linguistique, par laquelle est généralement décrit le rôle des enseignantes rencontrées. Ce rôle consiste à accompagner l' "engagement volontaire » des signataires-stagiaires de ce dispositif dans la réalisation active de leur projet de FL exigé, un projet que ces apprenant-e-s sont censé-e-s reconnaître de fait, en tant qu'il est le reflet d'un engagement prétendument choisi, ce dont atteste la signature du CAI.

19 Aussi, bien que des enseignantes nuancent sa capacité à garantir l'autonomie des signataires-stagiaires, au regard de son faible volume horaire ou parce qu'il n'est pas assez individualisé, le VL du CAI est décrit comme un espace censé permettre aux 
apprenant-e-s d'incorporer des règles légitimes, notamment linguistiques, nécessaires pour devenir des sujets autonomes, responsables et capables de s'adapter à la société française. Toutefois, en même temps qu'elle est décrite comme un objectif central et transversal de ce dispositif, nos enquêtes de terrain ont montré que l'évocation de cette autonomie est le lieu d'une exigence différenciée selon les sexes, révélatrice de l'inscription et de la réactualisation des rapports sociaux (Falquet, Lada \& Rabaud 2006).

\section{L'autonomie, une exigence différenciée selon les sexes : «c'est la mère qui emmène l'enfant à l'école »}

(1) / on a eu des cas (...) où on s'est fait insulter par des maris (rires) / parce que le fait que la formation soit pas purement linguistique / qu'il est inclus dans la formation l'autonomie de la femme notamment / c'est inclus dans la formation / qu'elles soient capables de se débrouiller seules dans la ville par exemple / (Sandrine.23.1)

(3) / et ces femmes / on a l'impression qu'elles sont / qu'elles ont toujours été accompagnées dans leur pays / c'est-à-dire que c'est souvent le mari qui faisait les démarches / en France c'est les enfants qui prennent le relais / (Diane. 11.6)

Puis, partant de ce qu'elle semble considérer comme un défaut d'autonomie, Diane réaffirme l'importance de la FL CAI :

(4) / et je pense que cette formation linguistique leur permet / pour celles qui l'acceptent / d'être autonomes (Diane. 11.6)

Et elle précise le sens qu'elle attribue au vocable autonomie : 
(5) / ça veut dire / c'est la mère qui emmène l'enfant à l'école / c'est pas l'enfant qui est là et sa mère est à côté pour faire semblant de l'emmener à l'école / donc y a / (...) / y a un début / (...) / d'autonomie pour ces femmes / certaines l'acceptent et sont conscientes que c'est une aubaine / d'autres préfèrent / dire voilà / (...) de toute façon / c'est pas moi qui fait les démarches / c'est mon mari / c'est mes enfants qui me servent d'interprètes / donc moi j'essaye de leur expliquer souvent / que les enfants interprètes c'est bien / mais c'est pas le rôle des enfants / et que c'est pas un service qu'on leur rend que d'être toujours / voilà / assistées / (Diane. 11.6)

Le contrat d'accueil et d'intégration est représentatif des dispositifs qui substituent la logique de l'usager-bénéficiaire à celle de l'usager-acteur-actif. Ces derniers se mettent en place autour de la remise en cause des formes de solidarité induites par d'anciens dispositifs, perçus comme des lieux potentiels de déresponsabilisation des individus et de production d'une assistance néfaste ${ }^{11}$.

La rhétorique des méfaits de l'assistance est particulièrement observable dans les propos de Diane qui décrit la FL CAI comme une étape permettant de contrer certaines formes de dépendance et d'assistance, pour l'autonomie des femmes. À cet endroit, ce principe apparait comme une capacité ou une qualité des stagiaires femmes à être dans un processus de relation à soi et aux autres qui permet, par l'appropriation du français, ainsi que des normes explicites et implicites nécessaires pour demeurer en France, de se libérer d'une forme de dépendance envisagée comme spécifique ou particulière.

Par ailleurs, tandis que la formation linguistique est décrite comme une aubaine pour les femmes qui l'acceptent, l'autonomie qu'elle est censée conférer est le lieu d'une exigence de séparation adressée à ces dernières, souvent décrites comme des figures de dominées archétypales, déjà-assistées par leurs conjoints, figures de dominants archétypaux. Enfin, cet idéal est convoqué à travers une figure et une position sociale en outre dépréciée dans les entretiens, celle de mère au foyer. À cet endroit, l'évocation de l'autonomie met en exergue une consubstantialité située des rapports sociaux, car si les femmes stagiaires apparaissent comme déjà-dépendantes, ce n'est sans doute pas parce qu'elles ne parlent pas, ou pas assez, la langue française, mais parce qu'elles sont des femmes, et des femmes racisées de surcroît (Guillaumin 2002).

Cette rhétorique de l'autonomie s'apparente à une sorte d'exhortation à se « délivrer de tout lien » (Hache 2007 : 53). Et, conformément à l'inédite vigueur qui impulse la mise en place du CAI, à partir de l'idée qu'il est nécessaire d'« en finir avec l'image "sociale", (...) à la fois misérabiliste et contestataire, des immigrés " pour " en montrer le visage de la réussite " (Fassin \& Mazouz 2007: 740), ce principe peut induire une forme de dévalorisation de certains liens d'interdépendance - principalement vis-à-vis des conjoints et des enfants - pour offrir un point de vue capacitaire et non victimaire de l'immigration et des immigré-e-s. Mais, simultanément, il masque des dépendances, des interdépendances et des incapacités intrinsèquement liées à l'émigration et à l'immigration, à la consubstantialité des rapports sociaux, et à la prégnance d'une division du travail au centre de l'infusion de l'autonomie, car c'est la mère qui emmène l'enfant à l'école (...). 


\section{Entre « le monde du travail » et « leur communauté » : autonomie, rapports sociaux et division du travail} souvent une sorte de continuité des prérogatives assignées aux femmes et aux hommes, lesquelles prérogatives sont associées à des qualités attendues de ces dernièr-e-s, dépassant le cadre prescriptif de ce dispositif. À cet égard, les propos de Sandrine semblent révélateurs d'un paradoxe qui accompagne le plébiscite de l'autonomie. Alors que ce dernier s'adresse prioritairement aux femmes stagiaires ${ }^{12}$ et qu'il est exigé d'elles qu'elles en adoptent les signes intérieurs et extérieurs pour se libérer d'une forme de dépendance spécifique et favoriser un meilleur management de soi-même, la réalisation de cette autonomie semble souvent limitée à la sphère domestique et au rôle de femme ou de mère au foyer.

En outre, cet extrait d'entretien met en relief une sorte de déjà-illégitimité de l'entresoi de ces femmes, visible à travers l'emploi différencié des termes le monde du travail, exclusivement mis en lien avec les hommes, et leur communauté, strictement mis en lien avec les femmes. Dans ce contexte discursif, la référence semble se situer autour du terme le monde du travail, tandis que l'expression leur communauté attire l'attention sur une altérité différenciée et racisée. Toutes les communautés ne se valent pas. Comme le montrent de nombreux travaux, les pratiques d'entre-soi (ou de communautarisme) de groupes considérés comme légitimes, prônant à la fois un individualisme universaliste et un collectivisme de classe séparatiste, ne sont généralement pas abordées d'un point de vue polémique, voire dangereux (Chevalier 2002). Alors que le mot communauté peut, par ailleurs, évoquer des formes de solidarité nécessaires pour faire société, 
l'entre-soi qu'il suppose ici apparait comme illégitime, non pas parce qu'il est un entresoi, mais parce qu'il est incarné par des femmes racisées. Ceci, faisant entrevoir les médiations autour desquelles peuvent s'articuler et s'actualiser les rapports sociaux asymétriques qui produisent et/ou réitèrent les assignations, par des processus, notamment discursifs, de différenciation et de hiérarchisation.

\section{"Des cours de français pour être dans la vie de leur enfant (...) et ne pas être sous la coupe de leur mari » : de l'articulation à l'actualisation des rapports sociaux}

Dans le cadre de nos enquêtes, la dimension hiérarchisée et hiérarchisante des discours et des actions relatives à l'autonomie et aux langues est souvent masquée par une rhétorique située de l'égalité entre les sexes, comme trait émancipateur de la société française, ce qu'illustrent les propos de Camille à travers les liens de corrélation qu'elle établit entre appropriation linguistique et autonomie :

(7) / je pense que c'est bien que les gens qui (...) ne parlent pas français puissent avoir des cours de français / je pense que c'est (...) / très important qu'ils réussissent à avoir une certaine autonomie dans un pays où ils vont vivre / je pense aussi que c'est le seul moyen pour certaines femmes de réussir à sortir de leur cocon familial (...) / ben y'en a quand même certaines qui ont des pressions communautaires et donc c'est un peu le moyen qu'elles ont pour voir un petit peu autre chose et puis (...) se familiariser aussi un petit peu avec (...) les coutumes françaises puisqu'on leur parle de ça aussi / (Camille.8)

Cet énoncé débute par l'emploi du général (les gens) à partir duquel se décline le féminin (les femmes), en annexe de l'argument de référence. Et, partant des pressions communautaires que subiraient certaines femmes, Camille décrit la FL CAI comme le seul moyen qui permettrait à celles-ci de sortir de leur cocon familial. Lui demandant d'expliciter le sens qu'elle attribue au terme autonomie, l'enseignante répond:

(8) / juste le fait de parler / de pouvoir s'exprimer donc de pouvoir / je sais pas / aller à la mairie / de pouvoir remplir un chèque / de pouvoir discuter avec les instit' de leurs enfants / (...) / là je parle surtout des femmes / parce que bon on a plutôt des femmes / avoir la possibilité d'être vraiment dans la vie de leur enfant / de pouvoir l'accompagner / d'avoir la possibilité / de ne pas être complètement enfermée et sous la coupe de leur mari / et pouvoir un petit peu s'émanciper / » (Camille.8.1)

Ces propos réitèrent l'importance de se garder de penser l'autonomie comme une injonction à l'agir indifférenciée et mettent en exergue la manière dont les rapports sociaux de sexe s'articulent avec les rapports sociaux de race, desquels résulte et qu'actualise la division sexuelle et raciale du travail. Après avoir établi un inventaire des activités ou des valeurs associées à l'autonomie, l'enseignante, évoque le cas des femmes stagiaires, et semble alors limiter les bénéfices de ce principe à leur statut et rôle de mères, d'épouses et à la sphère domestique, suggérant ainsi que, sans l'autonomie que confère la FL CAI, ces femmes pourraient ne pas être vraiment dans la vie de leur enfant. 
$35 \mathrm{Au}$ centre d'un mode de gouvernement de soi et de l'autre, et de nouvelles conflictualités sociales en constante recomposition, la mise en lien de l'obligation linguistique et de l'autonomie fait entrevoir la façon dont s'articulent et s'actualisent les rapports sociaux dans le cadre de ce dispositif de contrôle et de sécurité. Lorsqu'elle s'adresse aux hommes stagiaires, l'autonomie que confère la FL CAI est prioritairement définie par et pour les critères du monde du travail (cf. Sandrine en (6)), de la sphère productive, marchande et légitime. Et, lorsque ce principe s'adresse aux femmes stagiaires, elle est prioritairement définie par et pour la sphère reproductive, privée et domestique. Autrement dit, l'autonomie des hommes passe par la langue et elle est nécessaire pour figurer dans la sphère productive et marchande, au rôle exigé de travailleur-salarié, et l'autonomie des femmes passe également par la langue, mais paraît d'abord nécessaire pour figurer dans la sphère reproductive, au rôle tout aussi exigé de mère ou de femme au foyer, et pour s'autonomiser, soit s'éloigner de leurs conjoints. À ce titre, ces extraits d'entretiens attirent également l'attention sur une fausse alternative entre anti-sexisme et anti-racisme. Or, ce dilemme n'est pensable que dans la mesure où il est considéré que le sexisme et le racisme concernent deux groupes distincts. Outre son impossibilité empirique, une telle proposition implique également un dilemme et un conflit à l'intérieur des groupes qu'elle vise. De plus, comme le rappelle Delphy, «il n'existe pas de panneau annonçant : ici vous quittez le système patriarcal pour entrer dans le système raciste » $(2008: 212)$. Ces deux systèmes se combinent dans un même temps et un même espace pour les individus, en ayant en commun l'assignation des groupes sociaux à une place sociale, selon des processus de différenciation et de hiérarchisation qui les constituent. Ces rapports de pouvoirs relatifs au sexisme et au racisme concrétisent en même temps un accès différencié aux ressources matérielles, aux espaces de pouvoir et à la production des savoirs, impactant le registre de la classe (Palomares \& Testenoire $2010: 16)$.

Ce clivage des assignations est également accentué par la tension politique instituée entre immigration choisie (valorisée) et immigration subie (dévalorisée), la dernière catégorie rassemblant celles et ceux qui n'ont pas été choisi-e-s pour des raisons économiques. Renforçant l'assimilation des compétences linguistiques exigées à des compétences professionnelles, à partir d'une logique de responsabilisation où l'individu est prioritairement pensé à travers son employabilité (Heller 2011: 22), cette binarisation de l'immigration rappelle combien la présence légitime des immigré-e-s demeure liée au travail (Sayad 2006). D'ailleurs, le CAI rend inopérantes les catégories d'immigré-e-s au profit de celles de signataires et de stagiaires. Si ces termes sont neutres du point de vue du sexe et de l'origine, ils le sont moins du point de vue de leur référence économique ou utilitariste. Enfin, en prenant appui sur d'autres dialectiques (modernité/tradition, ici/là-bas, eux/nous, etc.) qu'elle actualise, cette mise en opposition de l'immigration renforce l'altérisation des immigré-e-s (Moujoud 2008).

\section{Quand ne pas pouvoir dire les choses est surtout handicapant pour une femme : l'intime pour définir l'autonomie}

Si l'appropriation de la langue légitime est envisagée comme un levier pour l'autonomie individuelle, elle n'est pas exigée par tout-e-s les stagiaires ou pour les mêmes raisons : 
(9) $\angle \mathrm{y}$ a les personnes qui parlent que leur langue chez eux / même au travail ça peut arriver qu'ils parlent que leur langue / (...) / ce qui me dérange aussi c'est / enfin / quand ils vont chez le médecin / avec une personne qui peut leur traduire ce qu'ils veulent dire / et qu'ils puissent pas dire les choses d'eux-mêmes / je trouve ça très (...) / handicapant / surtout pour une femme / (Nathalie.25.2) enseignantes vis-à-vis de l'appropriation linguistique, faisant entrevoir certains antagonismes autour desquels se forme et se transforme le principe d'autonomie, audelà de ce dispositif prescriptif. Demandant à Nathalie d'expliciter les raisons pour lesquelles ce phénomène serait davantage handicapant pour une femme que pour un homme, elle répond :

(10) / que tu puisses pas exprimer ce que tu ressens / tes problèmes / et que t'as besoin de quelqu'un / du coup / rien n'est personnel finalement / tu partages tout avec ta famille / et tout avec ton conjoint / ou avec ta cousine / ou avec ta sœur / handicapant / c'est peut-être pas le terme / mais en tout cas / ça te valorise pas / (Nathalie.25.2)

À l'endroit de ce dispositif argumentaire qui s'adosse sur le registre sémantique de l'intériorité, du ressenti, des besoins individuels et du discursif pour exprimer l'importance de l'indépendance personnelle des femmes stagiaires, indépendance et autonomie étant souvent corrélées, ces femmes semblent assignées à la position de personnes qui auraient un besoin spécifique d'exprimer leur ressenti et leurs problèmes.

Mettant l'accent sur l'important processus occidental d'individuation des trajectoires de vie tel qu'il a été amorcé à partir de la seconde moitié $d u$ xx ${ }^{\text {ème }}$ siècle, ces propos rappellent la centralité de la dimension individuelle et intime comme lieu de définition actuelle de l'autonomie. Dans ce cadre, l'exigence d'autonomie est fréquemment médiatisée par une obligation, directe ou indirecte, d'être capable de se détacher de l'autre pour accomplir des démarches seul-e :

(11) / je me rappelle d'une stagiaire / (...) / c'était les / la période de Noël / (...) / et je demandais à chaque stagiaire de / de faire un vœu / et le vœu d'une femme nous a beaucoup surpris / c'était de venir seule en formation / son vœu c'était de / de pas être accompagnée justement / d'être autonome et de pouvoir venir toute seule en cours / (Diane. 11.7)

Comme le propose Ehrenberg, en remettant en cause les approches individualistes de l'autonomie prises dans la dichotomie individu/société, cette perspective de l'autonomie individuelle peut induire une sorte de «relation causative à soi » (2005: 205). Elle laisse à penser qu'un individu peut se fonder lui-même et produire « tout seul, subjectivement et grâce à sa capacité réflexive, le lien social dans ses interactions avec d'autres sujets » (2005: 202). Or, dire que l'autonomie se fonde ou s'impose désormais à partir du registre de l'intime n'en fait pas moins une règle socialement instituée et imposée, dont la forme ne dépend pas directement des aspirations intimes des individus. Ce principe ne relève pas d'un idéal que chacun-e peut actualiser selon ses propres désirs ou capacités, mais d'une norme sociale qui s'impose institutionnellement aux individus. Si elle s'adresse prioritairement à des individualités, l'autonomie est instituée comme une contrainte à incorporer en dehors 
de ces mêmes individualités. Quand bien même cet idéal serait invoqué comme une mobilisation subjective et individuelle, il appartient au registre idéologique. Il est socialement institué et standardisé, donc un lieu potentiel d'instrumentation des parcours de vie et de réaffirmation des différences et des hiérarchies sociales, notamment linguistiques. Enfin, cette rhétorique de l'autonomie individuelle, conforme au modèle de l'égalité des chances et à l'ordre contractuel (Dubet 2010 : 92), peut avoir pour conséquence d'affaiblir les solidarités et les capacités à se penser collectivement, ignorant les processus de mobilité collective qui ont longtemps été des leviers pour l'intégration et pour l'autonomie des immigré-e-s.

\section{Les vertus de l'obligation pour l'autonomie des femmes : quand ne pas avoir le choix est une bonne chose}

Outre le caractère positif attribué à la formation linguistique CAI, c'est l'obligation de formation, elle-même, qui est plébiscitée par des enseignantes rencontrées :

(12) / on va dire que la formation linguistique leur permet de gagner en autonomie / même si / c'est aussi (...) toute une démarche dans leur esprit à faire / c'est vouloir aussi être autonome / et on voit que les (...) / la démarche qu'elles font déjà de venir régulièrement / donc de s'organiser à la maison / au niveau (...) / préparation des repas / au niveau des enfants / donc je trouve que c'est déjà une / une forme d'autonomie pour elles (...) / c'est un début (...) / bon la formation étant obligatoire / je pense que c'est une bonne chose / elles ont pas le choix / (...) / elles commencent à voler un petit peu de leurs propres ailes (...) / la formation est intensive / (...) / prendre le bus toutes seules / déjà la première fois elles sont accompagnées mais très vite elles prennent le bus toutes seules / le métro / elles commencent à se repérer dans Rennes / donc voilà / l'autonomie (...) / elle se fait avec la formation mais aussi avec tout ce que la formation entraine / comme autonomie / (Diane. 11.6)

Ces propos permettent de saisir certains antagonismes à partir desquels peut être légitimé le caractère contraignant et obligatoire de la FL CAI, au nom d'une version anti-sexiste située. Pour reprendre une formulation de Dubet : "Dans le monde idéal des chances, "vouloir, c'est pouvoir".» (2010: 81). Ainsi, même si Diane envisage que l'autonomie gagnée par la FL CAI, et ce qu'elle entraine, représente toute une démarche de l'esprit à faire, et qu'il est nécessaire de vouloir être autonome pour faire advenir cette autonomie, soit qu'elle est avant tout une aspiration qui «se prouve en s'éprouvant " (Foessel 2011 : 351), l'enseignante considère, paradoxalement, que c'est une bonne chose que les femmes stagiaires n'aient pas le choix. C'est au nom des bienfaits et des bénéfices qu'il entraine, notamment en termes d'autonomie, que le caractère obligatoire et contraignant de cette prescription linguistique est décrit comme bénéfique, lorsqu'il implique les femmes stagiaires. À travers ce glissement de l'aspiration à la condition (Ehrenberg 2011: 562), l'autonomie apparait comme une sorte de propriété des stagiaires à être ou à ne pas être autonomes ou comme le lieu de possession de quelque chose d'assez sécurisant pour se sentir libre d'avoir des projets (Dubet $2010: 104$ ). Formulé ainsi, cet idéal peut être un leurre qui invite à s'affranchir de l'autre, à ne rien lui devoir et à ne rien lui donner en retour.

Cette appréhension de l'autonomie est étroitement liée au soubassement contractuel du CAI, car, si l'autonomie linguistique est une exigence, le contrat est l'instrument qui 
assure et actualise cette exigence. Plus tôt, lors de mon échange avec Diane, je lui demandais si, selon elle, la forme contractuelle du CAI apportait quelque chose de positif en termes d'accueil ou d'intégration. L'enseignante répond par l'affirmatif :

(13) / des choses positives oui (...) / y a un public / surtout un public de femmes qui est resté pendant des années en France sans parler un mot de français / et ça c'est pas normal / donc ce contrat il a ce bon côté où il oblige / y a des hommes (...) / qui vont pas facilement laisser venir leur femme en cours / qui nous posent des conditions / elle ne viendra que le matin ou elle ne viendra que l'après-midi / (...) / y a toujours des explications de la part de l'homme / on prend pas en compte la femme / si la femme elle a envie de venir / si elle a envie de sortir / ou le mari qui l'attend à la sortie des cours parce qu'elle doit rentrer directement à la maison / et tout ça je pense que ce contrat il le brise un peu parce que l'homme il peut pas être là $\angle$ tous les jours / le matin / le midi / la surveiller / donc la femme gagne quelque part une petite autonomie / » (Diane. 8.1) d'orienter les discours et les actions sur le respect des procédures, l'engagement réciproque, l'égalité des parties, la liberté de contracter, l'autonomie de la volonté, etc. et par conséquent, de masquer son caractère unilatéral et contraignant, ainsi que l'asymétrie des relations sociales qu'il est susceptible d'induire. En effet, le contrat d'accueil et d'intégration ainsi que son volet linguistique n'impliquent ni réciprocité des obligations ni autonomie de la volonté, car « seul l'étranger prend des engagements d'une part et qu'il n'a d'autre choix que de signer le contrat d'autre part. » (Lochak 2011: s.p.)

\section{Le contrat d'accueil et d'intégration entre égalité et inégalités}

L'exigence d'autonomie est au centre des discours qui préfigurent le fonctionnement $\mathrm{du}$ volet linguistique du contrat d'accueil et d'intégration. Mais cette exigence renseigne sur une forme de figuration sociale légitime attendue des immigré-e-s primoarrivant-e-s en France qui dépasse le cadre prescriptif de ce dispositif d'offre-obligation de formation linguistique, faisant entrevoir la dimension dynamique et antagonique des relations et des pratiques sociales, leur caractère hiérarchisé et hiérarchisant.

La maitrise de la langue française est décrite comme une nécessité pour l'autonomie individuelle et pour l'égalité entre les sexes. En même temps, c'est à l'endroit de cette exigence faite aux femmes stagiaires de s'affranchir des dépendances, susceptibles de

GLAD!, 01 | 2016 
faire obstacle à leur autonomie individuelle, principalement vis-à-vis de leur conjoint, qu'apparait la part processuelle des rapports de pouvoir inhérents à ce dispositif prescriptif.

L'autonomie a «l'autorité d'une règle » (Ehrenberg, Mingasson \& Vulbeau 2005 : 115). De ce fait, son imposition fait partie intégrante des rapports de pouvoir. Cette imposition s'exerce non pas en dictant aux stagiaires ce qu'elles et ils ont à faire, mais ce qu'elles et ils sont, ce qui participera à les faire devenir durablement ce qu'elles et ils ont à être (Bourdieu 1982: 37). Et, c'est en tant que telle qu'elle s'infuse par des processus de différenciation et de hiérarchisation qui assignent et réaffirment des rôles et des statuts différenciés dont les effets sont potentiellement inégalitaires. L'invisibilisation de ces rapports sociaux est notamment garantie par la rhétorique de l'autonomie individuelle, qui fonctionne à l'image d'un opérateur idéal typique de libération par rapport aux liens d'interdépendance et de réification de l'individu tout puissant et, en même temps, souvent abandonné à lui-même.

Ces discours de (ou sur) l'autonomie sont à repositionner par rapport aux contraintes, aux jeux d'adhésion ou de distanciation vis-à-vis des institutions, donnant à voir des stratégies de recomposition avec les normes et les éventuelles tensions entre contrôle et autonomie.

51 En outre, "le continuum transnational des rapports sociaux de pouvoir " et le rôle prégnant du capitalisme dans les politiques migratoires ne peuvent être négligés (Falquet \& Rabaud 2008 : \$32), ces rapports sociaux étant conjointement fabriqués dans les sociétés d'origine et d'arrivée, via des cadres légaux et des accords bilatéraux qui les actualisent. À cet égard, rappelons pour conclure que l'exigence d'autonomie est à situer dans un mouvement de contractualisation de l'offre-obligation de formation linguistique à destination des immigré-e-s primo-arrivant-e-s en France, lui même adossé à un contexte d'individualisation des responsabilités et de contractualisation de l'action publique et des modes de gouvernance. Dans ce cadre, si la loi du 24 juillet 2006 est l'acte qui énonce et autorise objectivement le contrôle des trajectoires linguisticoculturelles, le contrat d'accueil et d'intégration permet de masquer la verticalité des relations qu'il induit pourtant, car, contrairement à la loi, il donne l'illusion que les signataires-stagiaires de ce dispositif peuvent tirer une plus-value d'une relation considérée comme négociée (Texier 2011:97). Cependant, comme la plupart des contrats, le CAI n'est pas strictement un contrat entre deux parties, mais plutôt un accord de deux parties à accepter les termes de principes définis extérieurement à eux. Le contrat d'accueil et d'intégration renvoie la demande sociale sur l'immigré-e qui doit individuellement solliciter son intégration et qui, dès lors, n'est plus considéré-e comme une personne qui subit une loi, mais comme une personne qui choisit de contracter un CAI, donnant ainsi l'illusion d'un ordre social dénué de rapports de pouvoir. Or, comme le rappelle Pateman: «Le contrat est le mode spécifiquement moderne par lequel sont créées des relations de subordination mais, du fait que la subordination civile prend sa source dans un contrat, elle est présentée comme une liberté. » (2010:170). 


\section{BIBLIOGRAPHIE}

ALBEROLA, Élodie \& DUBECHOT, Patrick. 2012. « La notion d'autonomie dans le travail social. L'exemple du RMI/RSA » Vie sociale 2012(1) : 145-156.

AMYOT, Jean-Jacques. 2012. «Vieillesse, contrôle social et idéologie sécuritaire. Entre autonomie et dépendance » Vie sociale 2012(1) : 127-143.

BOUQUET, Brigitte. 2012. « Analyse critique du concept de contrôle social. Intérêts, limites et risques » Vie sociale 2012(1) : 17-28.

BOURDIEU, Pierre. 1982. Ce que parler veut dire: L'économie des échanges linguistiques, Paris : Fayard.

CANUT, Cécile \& DUCHÊNE, Alexandre. 2011. «Introduction. Instrumentalisations politiques et économiques des langues : le plurilinguisme en question » Langage et société 136 : 5-12.

CATARINO, Christine. 2011. «Politiques migratoires et politiques d'emploi : la flexibilité sexuée en Europe » Cahiers du Genre 51 : 93-112.

CHEVALIER, Louis. 2002. Classes laborieuses et classes dangereuses à Paris pendant la première moitié du XIXe siècle. Paris : Perrin.

DELPHY, Christine. 2001. L'ennemi principal. 2, Penser le genre. Paris : Syllepse.

DELPHY, Christine. 2008. Classer, dominer : qui sont les « autres »? Paris : La Fabrique.

DUBET, François. 2010. Les places et les chances. Repenser la justice sociale. Paris : Seuil.

EHRENBERG, Alain. Juillet 2005. « Agir de soi-même » ESPRIT : 200-209. URL : http://jean-pierrevoyer.org/esprit-07-05-2-1.pdf

EHRENBERG, Alain. 2011. «La société du malaise. Une présentation pour un dialogue entre clinique et sociologie » Adolescence $77: 553$ - 570.

EHRENBERG, Alain, MINGASSON Lise \& VULBEAU Alain. 2005. «L'autonomie, nouvelle règle sociale » Informations sociales $126: 112-115$.

FALQUET, Jules, LADA Emmanuelle \& RABAUD Aude (éds.). 2006. (Ré)articulation des rapports sociaux de sexe, classe et "race" Repères historiques et contemporains, Cahiers du CEDREF 14.

FALQUET, Jules \& RABAUD, Aude. 2008. «Introduction » Cahiers du CEDREF 16 : 7-32. URL : http:// cedref.revues.org/571

FASSIN, Didier \& MAZOUZ, Sarah. 2007. « Qu'est-ce que devenir français ? La naturalisation comme rite d'institution républicain » Revue française de sociologie 48(4) : 723-750.

FASSIN, Éric. 2006. « La démocratie sexuelle et le conflit des civilisations » Multitudes 26 : 123-131.

FOESSEL, Michel. 2011. « Kant ou les vertus de l'autonomie », Études, Revue de culture contemporaine $414: 341-351$.

FOUCAULT, Michel. 2004. Sécurité, Territoire, Population. Cours au collège de France (1977-1978). Paris : Gallimard/Seuil.

GAUDIN, Jean-Pierre. 2007. Gouverner par contrat. Paris : Presses de Sciences Po.

GUILLAUMIN, Colette. 2002. L'idéologie raciste : Genèse et langage actuel. Paris : Gallimard.

HACHE, Émilie. 2007. « La responsabilité, une technique de gouvernementalité néolibérale ?» Raisons politiques $28: 49-65$. 
HELLER, Monica. 2011. « Du français comme "droit” au français comme "valeur ajoutée" : de la politique à l'économique au Canada » Langage et société 136 : 13-30.

JOUAN, Marlène. 2012. «L'autonomie, entre aspiration et injonction : un idéal ? » Vie sociale 2012(1) : 41-58.

KERGOAT, Danièle. 2004. « Division sexuelle du travail et rapports sociaux de sexe », in Dictionnaire critique du féminisme, HIRATA Helena, LABORIE Françoise, LE DOARÉ Hélène \& SENOTIER Danièle (éds.). Paris : PUF, 35-44.

KERGOAT, Danièle. 2009. « Dynamique et consubstantialité des rapports sociaux ", in Sexe, Race, Classe. Pour une épistémologie de la domination, DORLIN Elsa (éd.). Paris : PUF, 111-125.

LOCHAK, Danièle. 2011. « Le contrat d'accueil et d'intégration, une contractualisation biaisée pour une intégration alibi » Communication donnée à la Journée d'études La contractualisation du social, Université du Havre, le 7 avril 2011. URL : http://www.cirtai.org/projetuoh/lecon2--transcription.html

MOUJOUD, Nasima. 2008. «Effets de la migration sur le femmes et sur les rapports sociaux de sexe. Au-delà des visions binaires » Cahiers du CEDREF 16 : 57-79. URL : http://cedref.revues.org/ 577

PALOMARES, Elise \& TESTENOIRE, Armelle. 2010. « Indissociables et irréductibles : les rapports sociaux de genre, ethniques et de classe » L'Homme et la société 176-177(2) : 15-26.

PATEMAN, Carole. 2010. Le contrat sexuel. Paris : La Découverte.

PETIT, Christelle. 2012. « Acteurs sous contrôle : nouveau paradigme de l'action sociale. L'exemple du RSA » Vie sociale 2012(1) : 81-95.

PFEFFERKORN, Roland. 2012. Genre et rapports sociaux de sexe. Lausanne : Page deux.

SAYAD, Abdelmalek. 2006. L'Immigration ou les Paradoxes de l'altérité. Tome 1. L'illusion du provisoire. Paris : Raisons d'agir.

SCOTT, Joan W. 1988. « Genre : une catégorie utile d'analyse historique » Les Cahiers du GRIF, Le genre de l'histoire 37-38: 125-153.

TEXIER, Dominique. 2011. «Contractualisation. Contractualisation des rapports sociaux », in Adolescences contemporaines, TEXIER, Dominique. Paris : ÉRÈS, 96-105.

\section{ANNEXES}

\section{Références textuelles : Discours et déclarations publiques, études et rapports}

BESSON, Éric. 2010. Signature par Éric Besson du $500000^{e}$ Contrat d'Accueil et d'Intégration. Ministère de l'Immigration, de l'Intégration, de l'Identité nationale et du développement solidaire.

Code de l'entrée et du séjour des étrangers et du droit d'asile, art. L311-9. Modifié par Décret $\mathrm{n}^{\circ}$ 2009-331 du 25 mars 2009 - art. 5 (V). URL : https://www.legifrance.gouv.fr/ affichCode.do ;jsessionid =30AE4BE3B7A793BDE42406640738E4DB.tpdjo10v_3 ? idSectionTA $=$ LEGISCTA000006163227\&cidTexte $=$ LEGITEXT000006070158\&dateTexte $=20090724$ 
HAUT CONSEIL A L'INTEGRATION. 2003. Le contrat et l'intégration, Rapport du Haut Conseil à l'Intégration. URL : http://www.ladocumentationfrancaise.fr/var/storage/rapportspublics/044000033.pdf

Loi relative au contrôle de la validité des mariages, 14 novembre 2006, n² 2006-1376.

MARCHÉ OFII. 2012. « Cahier des clauses particulières ». n 13 FL. URL : http://www.criaquitaine.org/fileadmin/user_upload/mediatheque/Actions_de_formations/ Marche_OFII/Cahier_des_clauses_particulieres.pdf

MINISTÈRE DE L'EMPLOI, DE LA COHÉSION SOCIALE ET DU LOGEMENT. AGENCE NATIONALE DE L'ACCUEIL DES ÉTRANGERS ET DES MIGRATIONS. s.d. Bienvenue en France. Le Contrat d'Accueil et d'Intégration. URL : http://www.info-droits-etrangers.org/ menu-cote1/form-utiles/sejour/Contrat-accueil-et-integration.pdf [consulté le 31 octobre 2016]

PRIPI BRETAGNE. 2010. Programme Régional d'Intégration pour les Populations Immigrées Bretagne 2010-2012. Préfecture de l'Ille-et-Vilaine, Service Interministériel Immigration et Intégration.

SARKOZY, Nicolas. 2005. Déclaration de M. Nicolas Sarkozy, ministre de l'intérieur et de l'aménagement du territoire et président de l'UMP, sur les propositions de l'UMP en matière d'immigration, d'intégration et d'emploi, à l'Assemblée nationale le 9 juin 2005. URL : http:// discours.vie-publique.fr/notices/053001871.html

\section{NOTES}

1. Le questionnement général de cette recherche émerge de la mise en questionnement de deux contextes articulés : l'un relatif à la mise en fonctionnement du contrat d'accueil et d'intégration et de son volet linguistique en tant que dispositif des politiques de l'immigration et de l'intégration; et l'autre relatif à un contexte concomitant de mise en avant des questions relatives à l'égalité entre les sexes dans le débat public sur l'immigration et l'intégration.

2. Un dispositif issu d'une démarche entamée en 2007-2008, dont l'objectif est de recenser les offres d'apprentissage du français destinées au public étranger, sur le territoire de RennesMétropole.

3. Hors CAI : un parcours de formation linguistique destiné à un public étranger inéligible au CAI et/ou souhaitant poursuivre une formation linguistique après le CAI.

4. Article L311-9 du Code de l'entrée et du séjour des étrangers et du droit d'asile. Modifié par Décret n²009-331 du 25 mars 2009 - art. 5 (V). URL: https://www.legifrance.gouv.fr/ affichCode.do;jsessionid=30AE4BE3B7A793BDE42406640738E4DB.tpdjo10v_3?

idSectionTA=LEGISCTA000006163227\&cidTexte=LEGITEXT000006070158\&dateTexte $=20090724$

5. Intervention de N. Sarkozy, ministre d'État, ministre de l'Intérieur et de l'Aménagement du territoire, Président de l'Union pour un Mouvement Populaire, Convention pour un projet populaire - Une immigration choisie, une intégration réussie - Assemblée nationale, le 9 juin 2005. URL : http://discours.vie-publique.fr/notices/053001871.html

6. «Bienvenue en France». Le Contrat d'Accueil et d'Intégration. Ministère de l'emploi, de la cohésion sociale et du logement. Agence nationale de l'accueil des étrangers et des migrations, dans la partie intitulée «La France, un pays d'égalité ». URL : http://www.info-droits-etrangers.org/ menu-cote1/form-utiles/sejour/Contrat-accueil-et-integration.pdf 
7. Éric Besson, ministre de l'Immigration, de l'Intégration, de l'Identité nationale et du Développement solidaire, «Signature par Éric Besson du $500000^{\mathrm{e}}$ Contrat d'Accueil et d'Intégration », 2 mars $2010: 1-4$.

8. Cf. Marché OFII n 13 FL, « Cahier des clauses particulières », OFII 2012, p. 9.

9. En 2009, la répartition sexuée des signataires du CAI sur le territoire breton corrobore les observations nationales concernant l'augmentation de la part des femmes parmi la population immigrée, puisque $56,59 \%$ des signataires du CAI sont des femmes. En outre, parmi les 591 personnes soumis-e-s à une obligation de formation linguistique, ces dernières sont également majoritaires (68,7 \%). Source : PRIPI Bretagne 2010-2012 : 97-98.

10. Cf. Loi n 2006-1376 du 14 novembre 2006 relative au contrôle de la validité des mariages. À ce titre, toutes les femmes stagiaires interrogées ont contracté un CAI au titre du regroupement familial.

11. Comme l'illustre le passage du Revenu Minimum d'Insertion (RMI) au Revenu de Solidarité Active (RSA) qui subordonne toute aide à l'activité (Petit 2012 : 82).

12. Dans un même temps, une majorité des enseignantes s'excluent discursivement du jeu des rapports sociaux de sexe, ne se disant pas ou très rarement concernées par des rapports de pouvoir similaires. En effet, lorsqu'elles évoquent certains facteurs de vulnérabilité comme les contrats de travail facilement révocables qui concernent une majorité d'entre elles, les formes d'emploi vulnérables (cf. les remplacements), etc., ces formes potentiellement ségrégatives du travail sont principalement rapportées à des facteurs individuels et non à des effets de structure et d'organisation du travail, notamment liés à la mise en fonctionnement du CAI.

\section{RÉSUMÉS}

Partant d'une recherche qui a consisté à interroger l'actualisation du genre et des rapports sociaux à travers la mise en application du volet linguistique du contrat d'accueil et d'intégration à Rennes, cette contribution vise à interroger l'autonomie comme révélateur de logiques normatives et de rapports sociaux, circonscrits au fonctionnement de ce dispositif des politiques de l'immigration et de l'intégration, dans un contexte de multiplication des processus de suivi individualisés et contractualisés, privilégiant des mécanismes d'autonomisation individuelle. À partir d'enquêtes de terrain réalisées dans l'organisme agréé pour la formation linguistique contrat d'accueil et d'intégration (CAI) à Rennes, il s'agira plus particulièrement de décrire la manière dont apparait, dans ce contexte d'offre-obligation de formation linguistique, une forme d'injonction à l'autonomie par la langue, prioritairement adressée aux femmes stagiaires de ce dispositif. Ceci, illustrant la façon dont cet idéal de remise en cause des formes de contrôle social s'infuse par une invisibilisation des rapports de pouvoir qui lui sont pourtant inhérents.

Drawing on a research that questioned the reproduction of gender and power relations in the implementation of the language related components of the Accommodation and Integration Contract (CAI) in Rennes, this paper looks into the interpretation of « autonomy » as a principle revealing normative logics and situated social relations, that are delimited by the application process of this apparatus of French immigration policies. At a time when individualised and contractualised monitoring processes have been increasingly used to promote individualised autonomy/empowerment mechanisms, this fieldwork study, settled in a training organisation authorised to deliver the linguistic training of the CAI in Rennes, describes how these offered- 
required trainings reveal a form of injunction to autonomy, that is primarily directed at women trainees, through the practice of French. This thus illustrates how autonomy, as an ideal expected to challenge forms of social control, infuses the apparatus by making invisible the power relations that are inherent to it.

\section{INDEX}

Thèmes : Recherches

Keywords : autonomy, empowerment, gender, migrations, contractualisation, language

Mots-clés : autonomie, genre, migrations, contractualisation, langues

\section{AUTEUR}

\section{NADIA OUABDELMOUMEN}

Université Rennes 2, PREFics

Nadia Ouabdelmoumen est docteure en sciences du langage, Attachée Temporaire de Recherche au département communication à l'université Rennes 2 et membre associée du PREFics (EA 4246). Ses principaux domaines de recherche portent sur le genre, les migrations et les rapports de pouvoir, et visent plus particulièrement à mettre à l'épreuve du genre les récents dispositifs des politiques publiques liés aux migrations et à la conditionnalité linguistique qui s'appuient sur des logiques de contractualisation des rapports sociaux. 